\title{
Design and Development of the EMPOWER-SUSTAIN Prototype Web- Based Self-Management Mobile Apps: A Novel Empowerment Tool for Patients with Metabolic Syndrome
}

Maryam Hannah Daud ${ }^{1,2}$, Anis Safura Ramli1,2, Fakhrul Hazman Yusoff3, Nursuriati Jamil ${ }^{3}$, Suraya Abdul Razak ${ }^{1,2}$, Siti' Fatimah Badlishah Sham², Noorhida Baharudin'2, Mohamed Syarif Mohamed Yassin², Mohamad Rodi Isa4, Azlina Wati Nikmat ${ }^{5}$, Hapizah Nawawi ${ }^{1}$

${ }^{1}$ Institute of Pathology, Laboratory and Forensic Medicine (I-PPerForM), Universiti Teknologi MARA, Sungai Buloh Campus, Jalan Hospital, Sungai Buloh, Selangor; ${ }^{2}$ Department of Primary Care Medicine, Faculty of Medicine, Universiti Teknologi MARA, Selayang Campus, Batu Caves, Selangor; ${ }^{3}$ Department of Computer Science, Faculty of Computer \& Mathematical Sciences, Universiti Teknologi MARA, Shah Alam Campus, Selangor; ${ }^{4}$ Department of Population Health \& Preventive Medicine, Faculty of Medicine Universiti Teknologi MARA, Sungai Buloh Campus, Jalan Hospital, Sungai Buloh, Selangor; ${ }^{5}$ Department of Psychiatry, Faculty of Medicine, Universiti Teknologi MARA, Sungai Buloh Campus, Jalan Hospital, Sungai Buloh, Selangor.

\section{ABSTRACT}

Introduction: Given the proliferation of mobile phone users with accessibility to the internet, web-enabled mobile-based self-management intervention system is a potential solution to improve self-management behaviour among patients especially in the younger age groups.

Objective: This study aimed to design and develop the EMPOWER-SUSTAIN prototype webbased self-management mobile apps for patients with Metabolic Syndrome (MetS), to empower them with knowledge and skills to take charge of their health.

Methods: Iterative Model was chosen as the software development model for this study. Content from the newly revised EMPOWER-SUSTAIN Global Cardiovascular Risks SelfManagement Booklet $^{\odot}$ was evaluated for its suitability to be included in the prototype. A storyboard was designed to create the flow of prototype usage by Primary Care Physicians and patients during follow-up clinic and at home. In the pre-alpha stage, wireframe was designed to describe and visualize the user interface in static draft layouts based on the content and structure of information. Based on the wireframe, a mock prototype was designed to demonstrate the graphic representations of the content and function. Using the iterative model of the software development life cycle (SDLC), a working prototype was developed based on the mock prototype.

Results: High-fidelity mock-up static graphic diagrams demonstrating the content and function divided into eight sections were designed. The sections included My Profile, My Cardiovascular Risks, My Treatment Targets, My Check-Up, My Weight Management, My Smoking Habit, My Self-Management and My Medication. Based on the graphic diagrams, a working prototype of the EMPOWER-SUSTAIN web-based self-management mobile apps were developed using the iterative model of the SDLC.

Conclusion: The EMPOWER-SUSTAIN prototype web-based self-management was developed using a robust software development method. It is currently undergoing the alpha (utility) testing by medical experts and beta (usability) testing by patients with MetS.

Keywords: EMPOWER-SUSTAIN Prototype, Mobile Apps, Self-Management Support, Metabolic Syndrome 